\title{
Circulating tumor cells in hepatocellular carcinoma: a pilot study of detection, enumeration, and next-generation sequencing in cases and controls
}

Robin K Kelley ${ }^{1 *}$, Mark Jesus M Magbanua², Timothy M Butler ${ }^{3}$, Eric A Collisson², Jimmy Hwang ${ }^{2}$, Nikoletta Sidiropoulos ${ }^{4}$, Kimberley Evason ${ }^{5}$, Ryan M McWhirter ${ }^{2}$, Bilal Hameed ${ }^{6}$, Elizabeth M Wayne ${ }^{7}$, Francis $Y$ Yao $^{8}$, Alan P Venook ${ }^{1}$ and John W Park ${ }^{2}$

\begin{abstract}
Background: Circulating biomarkers are urgently needed in hepatocellular carcinoma (HCC). The aims of this study were to determine the feasibility of detecting and isolating circulating tumor cells (CTCS) in HCC patients using enrichment for epithelial cell adhesion molecule (EpCAM) expression, to examine their prognostic value, and to explore CTC-based DNA sequencing in metastatic HCC patients compared to a control cohort with non-malignant liver diseases (NMLD).

Methods: Whole blood was obtained from patients with metastatic HCC or NMLD. CTCs were enumerated by CellSearch then purified by immunomagnetic EpCAM enrichment and fluorescence-activated cell sorting. Targeted ion semiconductor sequencing was performed on whole genome-amplified DNA from CTCs, tumor specimens, and peripheral blood mononuclear cells (PBMC) when available.

Results: Twenty HCC and 10 NMLD patients enrolled. CTCs $\geq 2 / 7.5 \mathrm{~mL}$ were detected in $7 / 20$ (35\%, 95\% confidence interval: $12 \%, 60 \%) \mathrm{HCC}$ and $0 / 9$ eligible NMLD $(p=0.04)$. CTCs $\geq 1 / 7.5 \mathrm{~mL}$ was associated with alpha-fetoprotein $\geq 400 \mathrm{ng} / \mathrm{mL}(p=0.008)$ and vascular invasion $(p=0.009)$. Sequencing of CTC DNA identified characteristic HCC mutations. The proportion with $\geq 100 x$ coverage depth was lower in CTCs (43\%) than tumor or PBMC (87\%) $(p<0.025)$. Low frequency variants were higher in CTCS $(p<0.001)$.

Conclusions: CTCs are detectable by EpCAM enrichment in metastatic HCC, without confounding false positive background from NMLD. CTC detection was associated with poor prognostic factors. Sequencing of CTC DNA identified known HCC mutations but more low-frequency variants and lower coverage depth than FFPE or PBMC.
\end{abstract}

Keywords: Hepatocellular carcinoma (HCC), Circulating tumor cells (CTC), EpCAM, Sequencing

\section{Background}

Hepatocellular carcinoma (HCC) is a grim, heterogeneous disease with limited treatment options despite its enormous global impact as the third leading cause of cancer death worldwide [1]. Conventional liver imaging modalities for diagnosis and staging are imprecise and can result in underestimation of the true extent of disease, with microvascular invasion and multifocal tumors

\footnotetext{
* Correspondence: Katie.kelley@ucsf.edu

'Helen Diller Family Comprehensive Cancer Center and The Liver Center, University of California San Francisco (UCSF), 550 16th St., Box 3211, San Francisco, CA 94143, USA

Full list of author information is available at the end of the article
}

often identified incidentally at resection or transplant and associated with significantly poorer prognosis $[2,3]$. Translational research efforts to better understand the complex tumor biology of HCC, define biomarkers, and identify novel therapeutic targets are further limited by a scarcity of annotated, untreated tumor specimens, owing to the acceptance of radiographic diagnosis without tissue confirmation, the prevalence of liver-directed therapy before transplantation, and the risks associated with tumor biopsy in this population [4,5]. Non-invasive biomarkers for diagnosis and molecular characterization are urgently needed to overcome these pervasive challenges in HCC. 
Circulating tumor cells (CTCs) in the peripheral blood are a biomarker of poor prognosis in multiple epithelial tumor types [6,7]. The CellSearch System (Veridex LLC, Raritan, New Jersey, U.S.A) is an FDA-cleared device for CTC detection using enrichment for cells in the blood expressing the epithelial cell adhesion marker (EpCAM) [6]. The absolute numbers of CTCs detected and changes on therapy have been associated with survival and treatment response in breast, colon, and prostate cancers [8-13]. Multiple small studies have examined CTCs in patients with HCC using EpCAM- and nonEpCAM-based enrichment methods, with detection rates ranging from approximately $30 \%$ to over $80 \%$ depending on methodology and population [14-17]. As in other epithelial tumor types, the detection of CTCs by CellSearch correlates with poor prognosis in HCC cohorts, including increased recurrence risk after resection and shorter overall survival $[14,15]$.

In order to study CTCs as a biomarker in HCC, however, it is essential to establish that circulating epithelial cells in HCC populations are true tumor cells, rather than benign epithelial cells released into circulation as a consequence of the underlying inflammation or aberrant vasculature associated with liver disease. Though the detection of CTCs by CellSearch is extremely rare in healthy volunteers or patients with benign conditions $[6,10]$, there is limited data describing the incidence of circulating EpCAM-positive epithelial cells in the context of cirrhosis, viral hepatitis, or other causes of liver injury, conditions present in the majority of patients with HCC [14].

Beyond detection and enumeration, isolation of CTCs in cancer patients holds great promise as a "liquid biopsy", a non-invasive means of accessing real-time tumor tissue in the metastatic state for molecular profiling. Array comparative genomic hybridization has demonstrated concordance of characteristic copy number aberrations between CTC-derived DNA and archival primary tumor samples in breast, colon, prostate, and lung cancer [12,18-20]. Next-generation sequencing technologies now have the ability to sequence very small amounts of input DNA with high accuracy [21,22]. Illumina MiSeq technology can detect characteristic driver mutations in single CTCs derived from patients with metastatic colorectal cancer, concordant with the mutational profile of paired primary tumor specimens [18]. To date, the feasibility of efficient CTC isolation and molecular profiling, e.g. next-generation DNA sequencing, has not been reported in HCC.

We conducted this study to determine the proportion of metastatic HCC patients with detectable circulating EpCAM-positive epithelial cells using the CellSearch System, compared to a relevant control cohort of patients with liver disease, hypothesizing that circulating
EpCAM-positive cells are actual tumor cells rather than benign epithelial cells. To characterize their prognostic significance, CTC levels were examined for association with clinical covariates including alpha-fetoprotein (AFP) levels, the presence of vascular invasion, and overall survival. To explore the potential for CTCs to serve as a source of tumor DNA for genomic profiling in HCC, next-generation sequencing using a targeted cancer gene panel was performed using whole genome-amplified DNA derived from pooled purified CTCs, along with DNA from paired archival, paraffin-embedded tumor tissue and peripheral blood mononuclear cells when available.

\section{Methods}

\section{Study design}

This pilot study was a non-therapeutic, minimallyinvasive biomarker study. The trial was approved by the UCSF Committee on Human Research. All patients provided written informed consent for specimen collection and genetic testing of tumor and germline DNA. The study was conducted in accordance with the Declaration of Helsinki and Good Clinical Practice.

The primary endpoint was incidence of CTCs detected in metastatic HCC patients compared to a control cohort with NMLD. Secondary endpoints were enumeration of CTCs in each cohort, association with clinical and pathologic characteristics including alpha fetoprotein (AFP) level, tumor vascular invasion, and etiology of liver disease in the HCC cohort, and association with overall survival in the HCC cohort. An exploratory endpoint was to describe performance of and somatic mutations identified by next-generation sequencing of CTC whole-genome-amplified DNA along with paired tumor and germline DNA when available.

\section{Patient selection}

HCC patients were recruited at the UCSF Helen Diller Family Comprehensive Cancer Center. Principal inclusion criteria were: radiographic [4] or histologic diagnosis of American Joint Committee on Cancer (AJCC) stage IV HCC; $\geq 6$ weeks post biopsy, surgery, liverdirected interventions, or other invasive procedures; no prior systemic therapy or $\geq 4$ weeks since last dose of sorafenib or other systemic therapy for advanced HCC. Non-malignant liver disease (NMLD) control cohort patients were recruited at the UCSF Gastroenterology and Liver Disease Clinic. Principal inclusion criteria were: diagnosis of active hepatitis of any etiology plus clinical or pathologic diagnosis of cirrhosis or hepatic fibrosis (any stage); no evidence liver tumor on ultrasound or cross-sectional imaging within 6 months; AFP $\leq 20 \mathrm{ng} / \mathrm{mL}$ within 6 months; $\geq 6$ weeks post biopsy, surgery, or other invasive procedures; no prior history of $\mathrm{HCC}$. 


\section{Specimen collection}

Approximately $30 \mathrm{~mL}$ of whole blood was obtained from study subjects at a single time-point. For HCC patients with available archival tumor tissue from prior biopsy or resection, approximately five 10-micron sections of formalin-fixed, paraffin-embedded (FFPE) tumor along with a matching H\&E slide were collected from the pathology files of the University of California, San Francisco. Banked frozen aliquots of peripheral blood mononuclear cell (PBMC) were obtained when available from HCC cohort patients.

\section{Circulating tumor cell enumeration}

CTCs were isolated from $7.5 \mathrm{~mL}$ whole blood and enumerated using the CellSearch System (Veridex LLC, Raritan, NJ) [6-8]. Briefly, specific antibodies to EpCAM were used to enrich for epithelial cells. A mixture of fluorescently-labeled monoclonal antibodies to cytokeratin and the nuclear dye DAPI were used to select for nucleated, keratin-positive cells. CTCs were defined as nucleated, EpCAM-positive cells that stain positive for cytokeratin and negative for leukocyte common antigen, CD45 [6]. Labeled cells were enumerated using semiautomated fluorescence-based microscopy. Analysis was performed by a trained technician blinded to diagnosis (HCC versus NMLD).

\section{Immunoenrichment and fluorescence-activated cell sorting (IE/FACS)}

A novel EpCAM-based immunoenrichment (IE)/fluorescence-activated cell sorting (FACS) procedure has been developed to isolate purified CTCs without contamination from normal blood cells and has demonstrated correlation with CellSearch System CTC enumeration $[12,19,23]$. For patients found to have $>10$ CTCs in $7.5 \mathrm{~mL}$ of whole blood by CellSearch System, IE/FACS was then performed to isolate purified CTCs as has been previously described [12,24]. Briefly, approximately 15$20 \mathrm{~mL}$ of whole blood was incubated with immunomagnetic particles coated with two different monoclonal antibodies to EpCAM, one conjugated to magnetic particles and the other to a fluorophore. FACS was used to isolate nucleated, EpCAM-positive, CD45-negative cells.

\section{Whole genome amplification (WGA)}

A ligation-adaptor method of WGA was performed on whole cell lysates from pooled CTCs isolated by IE/ FACS using a GenomePlex whole genome amplification kit (WGA4, Sigma-Aldrich) according to the manufacturer's instructions $[12,25]$. DNA was randomly fragmented and converted to polymerase chain reaction (PCR)-amplifiable library molecules flanked by universal priming sites. PCR amplification of library molecules was performed using universal oligonucleotide primers.

\section{DNA extraction from tumor tissue and peripheral blood mononuclear cells (PBMC)}

Tumor-containing FFPE sections were identified and marked by a hepatopathologist (KE). DNA was extracted from FFPE sections as well as from banked PBMC using QIAmp kits (Qiagen) according to the manufacturer's instructions. DNA concentration was quantified using PicoGreen.

\section{Ion semiconductor NGS}

Sequencing of DNA extracted from CTCs, FFPE, and PBMC was performed by TMB in the Spellman Laboratory at Oregon Health Sciences University. From each sample, 10 ng DNA was PCR-amplified using AmpliSeq Cancer Panel Primer Pools and Library Kit 2.0 to generate 190 multiplexed amplicons (representing 46 cancer-related genes) [21]. Up to 11 barcoded samples were multiplexed on Ion 318 chips. Sequencing was performed on a Personal Genome Machine (PGM) sequencer (Ion Torrent) using the Ion PGM 200 sequencing kit. Torrent Suite software version 4.0.1 was employed to analyze read counts and quality. Variant Caller software version 4.0.1 identified variants. Coverage Analysis software version 4.0.1 determined target coverage. To minimize false positives, variants were required to have sequencing depth of at least 20x, an allele frequency of 5 percent, and not be present in any of the 3 PBMC samples sequenced. Variant calls were filtered against the Single Nucleotide Polymorphism Database (dbSNP) version 132, using the software ANNOVAR. Protein-altering variants were predicted by Mutation Assessor version 2 (http://mutationassessor.org).

\section{Statistical analysis}

Based upon the a priori hypothesis that approximately $50 \%$ of the HCC cohort and none of the NMLD cohort would have detectable CTCs by CellSearch, the planned sample size for this pilot study was 20 patients with metastatic HCC and 10 patients with NMLD, to permit estimation of proportion of detectable CTCs with 95\% confidence intervals $(\mathrm{CI})$ as $(0.30,0.70)$ in the $\mathrm{HCC}$ cohort and $(0.01,0.26)$ in the NMLD cohort. The incidence and number of detectable CTCs were analyzed using frequency and proportions with $95 \% \mathrm{CI}$ and compared between HCC and NMLD cohorts using the Wilcoxon-Kruskal-Wallis rank test. Cut-points of $\geq 1, \geq 2$, $\geq 3$, and $\geq 5 \mathrm{CTCs} / 7.5 \mathrm{~mL}$ were examined based upon published literature in HCC and other tumor types $[8,10,14,15]$. Wilcoxon-Kruskal-Wallis rank testing was also used to determine association between the presence of detectable CTCs by CellSearch System, AFP elevation using $\geq 400 \mathrm{ng} / \mathrm{mL}$ as an established prognostic cut-point [26,27], and the presence of vascular invasion (all binary variables). In the HCC cohort, overall survival was measured in months from date of CTC blood draw to the date of death 
with censoring at date of last known vital status if lost to follow-up. Kaplan-Meier methods were used to determine the impact of CTCs at each cut-point and conventional prognostic factors on overall survival. The CTC level, AFP value of $400 \mathrm{ng} / \mathrm{mL}$, and presence of macrovessel invasion were used to dichotomize for univariate analyses. The Child Pugh score and etiology of liver disease were also examined. A $p$ value of $<0.05$ was considered statisticallysignificant under log-rank tests. Sequencing coverage depth was compared between sample types using twotailed t-tests assuming unequal variance. Variant calls were reported descriptively due to small sample size.

\section{Results}

\section{Patient characteristics}

Twenty patients with a diagnosis of metastatic HCC (HCC cohort) and 10 patients with underlying nonmalignant liver disease without cancer (NMLD cohort) were prospectively enrolled between June 2011 and April 2012. All HCC patients were followed to date of death. Baseline patient characteristics are shown in Table 1. The median overall survival in the HCC cohort was 9.44 months from date of CTC blood draw. One NMLD cohort patient with HCV cirrhosis (Hep 25) was found to have a liver mass with adjacent portal vein thrombosis on a surveillance ultrasound after enrollment and was excluded based upon a suspected new diagnosis of HCC, resulting in 9 eligible patients in the NMLD cohort. The patient was subsequently lost to follow up. Figure 1 displays the study subject enrollment and samples tested.

\section{CTC detection and enumeration by CellSearch}

Figure 2 depicts the number of CTCs detected in each patient. At least 1 CTC per $7.5 \mathrm{~mL}$ was detected in 8 of 20 (40\%, 95\% CI: 17\%, 64\%) HCC patients and 1 of 9 (11\%, 95\% CI: $0,37 \%)$ eligible NMLD patients $(p=0.1$, Wilcoxon-Kruskal-Wallis rank test). At least 2 CTC per $7.5 \mathrm{~mL}$ were detected in 7 of 20 (35\%, 95\% CI: $12 \%$, 60\%) HCC patients and 0 of 9 eligible NMLD patients $(p=0.04$, Wilcoxon-Kruskal-Wallis rank test). Among the HCC cohort patients, at least 1 CTC per $7.5 \mathrm{~mL}$ was detected in 7 of $10(70 \%, 95 \%$ CI: $35 \%, 100 \%)$ with AFP $\geq$ $400 \mathrm{ng} / \mathrm{mL}$, versus 1 of 10 (10\%, 95\% CI: $0,33 \%)$ with AFP $<400 \mathrm{ng} / \mathrm{mL}(p=0.008)$. At least 1 CTC per $7.5 \mathrm{~mL}$ was detected in 8 of 13 (62\%, 95\% CI: 31\%, 92\%) with vascular invasion versus 0 of 7 without $(p=0.009)$ (Wilcoxon-Kruskal-Wallis rank tests). The NMLD control cohort patient Hep 25 who was removed for ineligibility (due to new liver mass with thrombosis consistent with HCC) was found to have 20 CTCs per $7.5 \mathrm{~mL}$ peripheral blood. Another NMLD cohort patient with alcoholic cirrhosis had 1 CTC detected per $7.5 \mathrm{~mL}$ peripheral blood. It is noteworthy that the single eligible NMLD control patient with detectable CTCs (1 in $7.5 \mathrm{~mL}$ ) subsequently
Table 1 Patient characteristics

\begin{tabular}{|c|c|c|}
\hline & $\begin{array}{l}\text { HCC cohort } \\
(n=20)\end{array}$ & $\begin{array}{l}\text { NMLD control } \\
\text { Cohort }(n=10)\end{array}$ \\
\hline Median age (range) (years) & $61.5(50-82)$ & 26-91 (53.5) \\
\hline Male/female $(n)$ & $20 / 0$ & $9 / 1$ \\
\hline \multicolumn{3}{|l|}{ Etiology of liver disease (\%) } \\
\hline HBV & 25 & 20 \\
\hline $\mathrm{HCV}$ & 45 & 60 \\
\hline Co-infection HBV + HCV & 10 & 0 \\
\hline $\mathrm{ETOH}$ & 5 & 10 \\
\hline NAFLD & 10 & 0 \\
\hline PSC & 0 & 10 \\
\hline Unknown & 5 & 0 \\
\hline \multicolumn{3}{|l|}{ Race/ethnicity (\%) } \\
\hline African-American & 5 & 10 \\
\hline Asian & 35 & 10 \\
\hline Caucasian & 55 & 70 \\
\hline Hispanic/Latino & 5 & 30 \\
\hline Non-Hispanic/Latino & 50 & 40 \\
\hline Native American & 5 & 0 \\
\hline Other/unknown & 0 & 10 \\
\hline \multicolumn{3}{|l|}{ Child Pugh score (\%) } \\
\hline A/B/C/unknown & $70 / 25 / 5 / 0$ & $30 / 30 / 30 / 10$ \\
\hline Median AFP (range) (ng/mL) & $492(3.8-587,134)$ & $5.5(1.7-17.2)$ \\
\hline BCLC score C $(\%)^{b}$ & 100 & N/A \\
\hline Vascular invasion (\%) & 65 & N/A \\
\hline Extrahepatic spread (\%) ${ }^{\mathrm{b}}$ & 100 & N/A \\
\hline Median overall survival (months) & 9.4 months & Not measured \\
\hline
\end{tabular}

Key: $\mathrm{HBV}=$ hepatitis $\mathrm{B}$ virus. $\mathrm{HCV}=$ hepatitis $\mathrm{C}$ virus. $\mathrm{ETOH}=$ alcohol. $\mathrm{NAFLD}=$ non-alcoholic fatty liver disease. $\mathrm{PSC}=$ primary sclerosing cholangitis. $\mathrm{BCLC}=$ Barcelona Clinic Liver Cancer. N/A = not applicable.

${ }^{a}$ Defined as HCV antibody positive plus either HBV surface antigen and/or core antibody positive.

${ }^{\mathrm{b}} \mathrm{BCLC} \mathrm{C}$ and presence of extrahepatic spread were required eligibility criteria for HCC cohort.

developed new infiltrative changes in the liver on a surveillance ultrasound, raising the possibility of underlying tumor though no formal HCC diagnosis was made before his death of complications of cirrhosis approximately 13 months after CTC blood draw.

The median overall survival (OS) in the HCC cohort was 9.4 months. Among HCC cohort patients with at least $1 \mathrm{CTC}$ per $7.5 \mathrm{~mL}$, the median OS was 2.8 months (95\% CI: $1.08,15.5)$, versus 11.3 months (95\% CI: 7.49, 12.9) for those without CTCs detected, although the difference was not statistically significant $(p=0.62$, LogRank test) (Figure 3). In univariate analysis of CTC levels and conventional prognostic factors (Table 2), none showed significant effect on overall survival, though analyses were limited by small sample sizes; no further multivariate analysis was performed. 


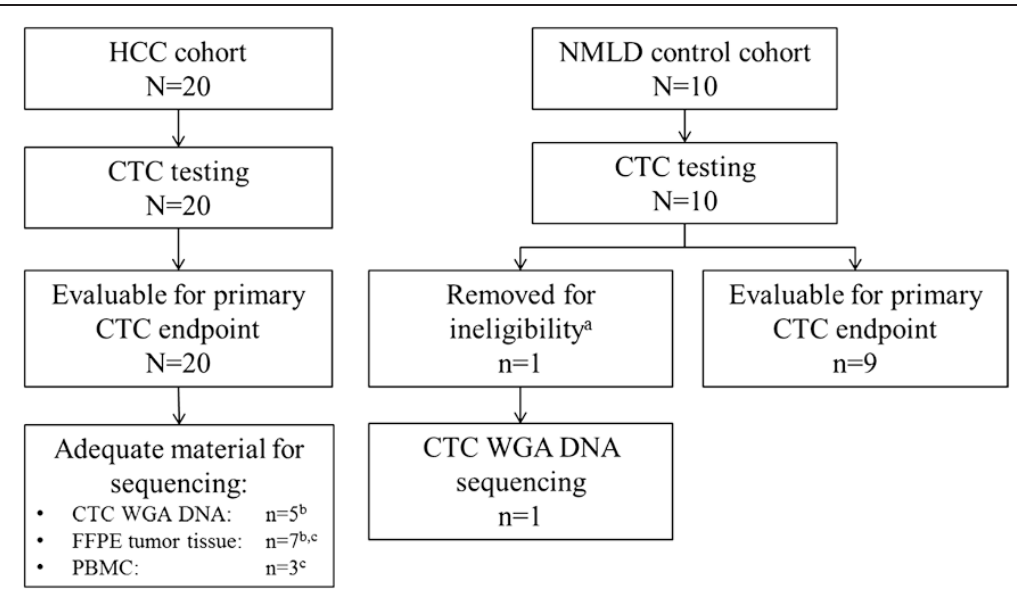

Figure 1 Study subject enrollment and samples tested. ${ }^{a}$ One patient enrolled to NMLD control cohort was removed for ineligibility due to new finding of liver mass with portal vein thrombosis on imaging after enrollment. CTC testing in this patient showed 20 CTCS per $7.5 \mathrm{~mL}$ peripheral blood. ${ }^{b}$ One sample each of CTC and FFPE did not yield sufficient DNA for sequencing. ${ }^{c} 4$ primary and 3 metastatic tumor FFPE samples were available from 7 of the HCC cohort cases. Paired CTC WGA DNA and FFPE tumor tissue were available in 2 cases, one of which also had PBMC available. Paired FFPE tumor tissue and PBMC were available from 2 additional cases.

\section{CTC isolation by IE/FACS}

Five patients in the HCC cohort showed greater than 10 CTC per $7.5 \mathrm{~mL}$ detected by CellSearch. CTCs were then isolated via IE/FACS performed on the remaining blood samples collected from these patients. IE/FACS was also performed on the specimen from Hep 25, the patient removed from the NMLD cohort for the finding of a liver mass with portal vein thrombosis. Absolute CTC counts by CellSearch and IE/FACS for these samples are provided in Additional file 1.

\section{CTC, PBMC, and FFPE sequencing performance}

Sequencing of adequate DNA samples from CTCs, FFPE tumor samples, and banked PBMC from the study cohort (Figure 1, Table 3) was performed. Paired FFPE tumor and/or PBMC from patients with adequate CTC DNA for sequencing were available in two cases; two additional cases with paired FFPE tumor and PBMC samples available without adequate CTC DNA also were analyzed from the HCC cohort (Figure 1). Sequencing performance according to sample type is displayed in Table 3. Sequencing performance data for FFPE tumor samples and banked PBMC (both a source of DNA not requiring WGA) were combined due to small sample sizes, for comparison to WGA DNA from CTCs (Table 3). The mean amplicon read depth was lower (2258 versus 2954, $p<0.01)$ and proportion of targeted bases with sequencing coverage of $\geq 100 \mathrm{x}$ was significantly lower in CTC samples (43\%) than in FFPE tumor plus PBMC samples $(87 \%)(p<$ $0.025)$, using two-tailed $t$-tests. The mean number of variant calls per sample was higher in CTC samples compared to FFPE samples ( 9 vs. $2, p<0.04$ ), though the mean frequency of individual variant alleles was significantly lower (36\% vs. $60 \%, p<0.001$ ) (two-tailed t-tests). Reproducibility of sequencing results was demonstrated by 3 samples run in duplicate (data not shown).

\section{Sequencing results: variants, SNPs and mutation calls}

Eighty-six variants overall, 58 of which were predicted to be protein-altering, were identified from all of the CTC
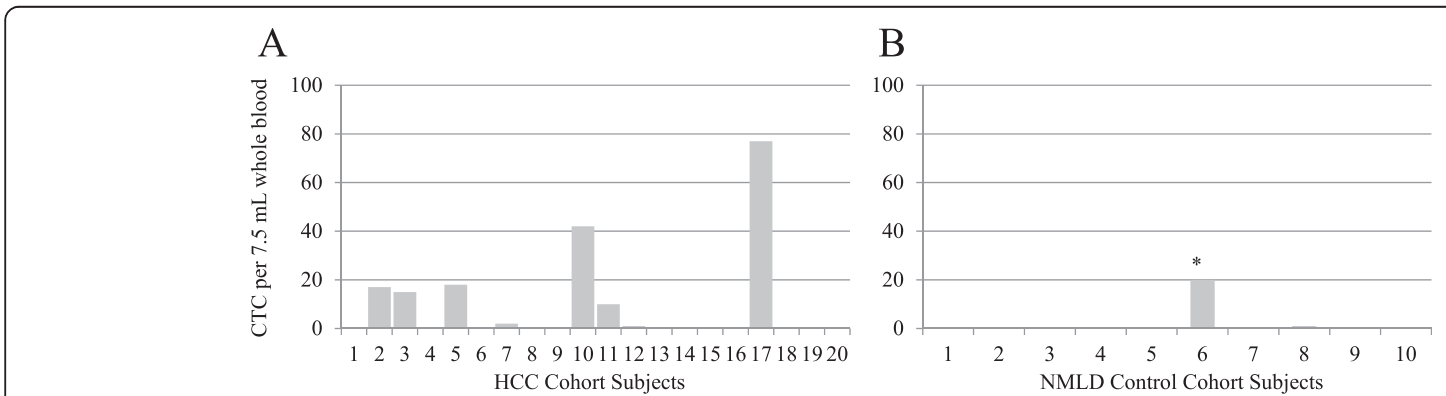

Figure 2 CTC detection and enumeration by CellSearch. Figure 2 depicts the CTC count per $7.5 \mathrm{~mL}$ whole blood by CellSearch in the HCC cohort (A) and NMLD control cohort (B). *One patient in NMLD cohort who was removed for ineligibility due to new liver mass with portal vein thrombosis was found to have 20 CTCs per $7.5 \mathrm{~mL}$ peripheral blood. 


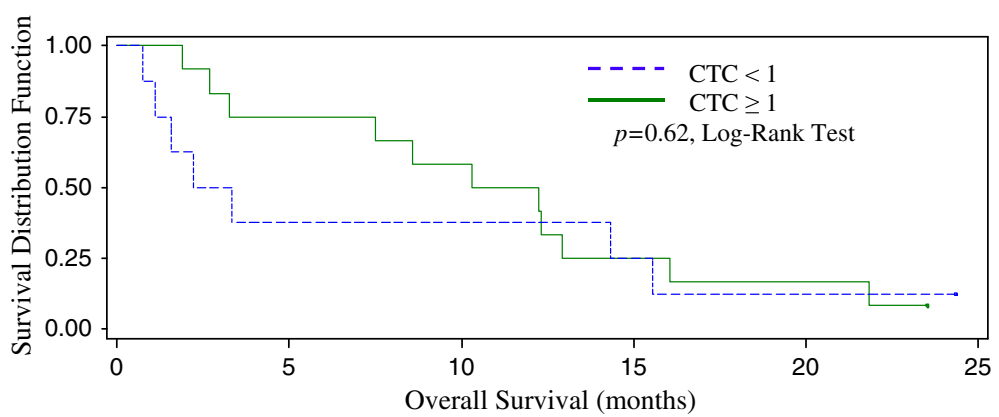

Figure 3 Kaplan-Meier survival curve in HCC cohort by CTC strata. Overall survival was measured from date of CTC blood draw to date of death. The median overall survival was 2.8 months in patients with $\mathrm{CTC} \geq 1 / 7.5 \mathrm{~mL}(95 \% \mathrm{Cl}: 1.08,15.5)$ versus 11.3 months in patients with CTC < $1 / 7.5 \mathrm{~mL}(95 \% \mathrm{Cl}: 7.49,12.9)$ though the difference was not statistically significant in this small sample ( $p=0.62$, Log-Rank test).

Table 2 Univariate analysis of CTC levels and conventional prognostic factors with overall survival

\begin{tabular}{|c|c|c|c|}
\hline HCC Cohort $(n=20)$ & $\begin{array}{l}\text { Mean overall survival } \\
\text { (months) (standard error) }\end{array}$ & $\begin{array}{l}\text { Median overall survival } \\
\text { (months) }(95 \% \mathrm{Cl})\end{array}$ & $p$ value (Log-Rank test) \\
\hline \multicolumn{4}{|l|}{$\overline{\mathrm{CTC}}$ per $7.5 \mathrm{~mL}$} \\
\hline$<1.0(n=12)$ & $10.96(1.95)$ & $11.29(2,69,16.06)$ & \\
\hline $1.0(n=8)$ & 8.49 (3.63) & $2.76(0.72,15.54)$ & 0.6179 \\
\hline$<2.0(n=13)$ & $10.37(1.89)$ & $10.32(3.25,12.91)$ & 0.8021 \\
\hline$\geq 2.0(n=7)$ & $9.23(4.11)$ & $2.20(0.72,15.54)$ & 0.8510 \\
\hline$<3.0(n=14)$ & $9.74(1.86)$ & $9.45(2.69,12.91)$ & \\
\hline$\geq 3.0(n=6)$ & $10.50(4.62)$ & $8.26(0.72,29.14)$ & \\
\hline \multicolumn{4}{|l|}{ Median AFP (ng/mL) } \\
\hline$<400(n=10)$ & $11.20(2.29)$ & $11.32(2.69,16.07)$ & 0.4058 \\
\hline$\geq 400(n=10)$ & $8.73(2.92)$ & $5.39(0.72,14.32)$ & \\
\hline \multicolumn{4}{|l|}{ Macrovessel invasion } \\
\hline No $(n=7)$ & $10.12(2.48)$ & $10.32(2.69,12.91)$ & 0.7493 \\
\hline Yes $(n=13)$ & $10.45(2.82)$ & $8.58(1.58,15.54)$ & \\
\hline \multicolumn{4}{|l|}{ Child Pugh score (\%) } \\
\hline $\mathrm{A}(n=14)$ & $10.69(1.87)$ & $11.32(2.20,15.54)$ & \\
\hline $\mathrm{B}(n=5)$ & $9.29(5.39)$ & $3.25(0.72,29.14)$ & 0.7181 \\
\hline$C(n=1)$ & I & & \\
\hline \multicolumn{4}{|c|}{ Etiology of liver disease (\%) } \\
\hline $\operatorname{HBV}(n=5)$ & $10.28(3.83)$ & $8.58(2.20,21.85)$ & \\
\hline $\operatorname{HCV}(n=9)$ & $10.41(1.96)$ & $12.62(1.91,15.54)$ & 0.9324 \\
\hline $\mathrm{HBV}+\mathrm{HCV}(n=2)$ & I & । & \\
\hline $\mathrm{ETOH}(n=1)$ & I & । & \\
\hline $\operatorname{NAFLD}(n=2)$ & I & । & \\
\hline Unknown $(n=1)$ & I & । & \\
\hline
\end{tabular}

Kaplan-Meier methods were used to determine the impact of CTC at each cut-point and conventional prognostic factors on overall survival. The CTC level, AFP value of $400 \mathrm{ng} / \mathrm{mL}$, and presence of macrovessel invasion were used to dichotomize for univariate analyses. The Child Pugh score and etiology of liver disease were also examined. A $p$ value of $<0.05$ was considered statistically-significant under log-rank tests. No factor showed significance in univariate analysis though analyses were limited due to small small sample sizes. Key: $\mathrm{Cl}=$ confidence interval. $\mathrm{ETOH}=$ alcohol. NAFLD = non-alcoholic fatty liver disease. $\mathrm{I}=$ sample size insufficient for analysis. 
Table 3 Sequencing performance by sample type

\begin{tabular}{llll}
\hline Sample type & CTC WGA DNA ( $\boldsymbol{n = 5 )}$ & $\begin{array}{l}\text { FFPE Tumor DNA }(\boldsymbol{n}=\mathbf{6}) \text { and } \\
\text { PBMC DNA }(\boldsymbol{n}=\mathbf{3})\left(\boldsymbol{n}=\mathbf{9} \text { total }^{\mathrm{a}}\right)\end{array}$ & $\boldsymbol{p}$ value (two-tailed t-test) \\
\hline Mean read length & $74 \mathrm{bp}$ & $76 \mathrm{bp}$ & $\mathrm{NS}$ \\
Mean mapped reads per sample & $653,878 \mathrm{bp}$ & $668,633 \mathrm{bp}$ & $\mathrm{NS}$ \\
Mean amplicon read depth (std. dev) & $2258(4389)$ & $2954(1379)$ & $p<0.01$ \\
Proportion with coverage > 20x & $50 \%$ & $97 \%$ & $p<0.0002$ \\
Proportion with coverage > 100x & $43 \%$ & $88 \%$ & $p<0.026$ \\
Mean non-synonymous variant calls per sample & 9 & $2^{\mathrm{b}}$ & $p<0.03$ \\
Mean variant allele frequency & $37 \%$ & $61 \%{ }^{\mathrm{b}}$ & $p<0.0001$ \\
\hline
\end{tabular}

${ }^{a}$ Data from FFPE and PBMC DNA samples were combined for sequencing performance analyses (but not for genotype analyses) due to small sample size and similar observed coverage. NS = not significant. ${ }^{\text {b } P B M C}$ samples (germline DNA) were excluded from variant analyses, $n=3$.

and FFPE tumor samples combined. Approximately 54\% were low-frequency (occurring in less than $10 \%$ of the individual sample), among which 93\% were from CTCderived DNA. Fifty-eight somatic, non-synonymous variants were called mutations if a matching mutation has been described in liver cancer, if the variant shared the same amino acid residue as a COSMIC mutation in any cancer type, and/or if the variant allele frequency was greater than $5 \%$ but the variant was not a known SNP and not present in any PBMC sample [28]. Frameshift mutations were excluded from analysis due to known limitations of ion semiconductor sequencing to accurately detect frameshift mutations. Characteristic mutations in HCC (TP53, PTEN) were identified in CTCderived DNA from two cases. Figure 4 displays a summary of the somatic, non-synonymous mutations identified in CTC and FFPE tumor samples combined. A listing of all somatic, non-synonymous mutations (excluding frameshift) detected according to sample type is provided in Additional file 2. In one $\mathrm{HCC}$ case with matched CTC, FFPE tumor, and PBMC DNA, 8 SNPs were present and concordant in both FFPE tumor and PBMC DNA; 5 of these (63\%) were detected in the CTC DNA. Neither was identified in the paired CTC DNA.

\section{Discussion}

The ability to detect and characterize malignant cells in circulation holds enormous promise as a biomarker in HCC, a grim cancer challenged by the inability of conventional noninvasive diagnostic and staging modalities to encompass its great clinical and biological heterogeneity, as well as by a scarcity of tumor tissue available for diagnostic or research purposes. In this study, at least one CTC was detected in $8 / 20(40 \%)$ of patients with metastatic HCC, compared to $1 / 9$ (11\%) of eligible NMLD patients using the CellSearch System. Though the cut-point of $\geq 1 \mathrm{CTC} / 7.5 \mathrm{~mL}$ did not achieve significance between the two groups, a cut-point of $\geq 2$ CTCs/ $7.5 \mathrm{~mL}$ was significant, positive in $7 / 20$ (35\%) $\mathrm{HCC}$ patients compared with none in the NMLD cohort ( $p=$ $0.04)$, consistent with prior reports $[14,15]$. The one eligible NMLD control patient with CTC count of 1 / $7.5 \mathrm{~mL}$ was subsequently found to have ultrasound findings suggestive of underlying tumor, although no formal HCC diagnosis was made, and thus he was not removed from the control cohort. Our findings confirm the limited existing data suggesting that circulating EpCAMpositive epithelial cells are rare in patients with nonmalignant liver diseases, and that EpCAM-positive cells in HCC patients are generally of tumor origin [14].

Corroborating the prognostic value of EpCAMpositive CTCs in other recent series [14,15], the detection of CTCs in the HCC cohort of this study was significantly associated with high AFP and the presence of vascular invasion, and there was a non-significant trend toward poorer overall survival in patients with detectable CTCs. These findings support the value of CTCs as a prognostic biomarker in metastatic HCC and suggest future potential roles for CTCs in treatment decisionmaking as well as for stratification in clinical research, which historically has been challenged by the great prognostic heterogeneity of this disease [29].

The unexpected finding of high CTC levels in a patient initially enrolled to the NMLD cohort, who subsequently was removed for ineligibility due to the finding of a new liver mass with vascular invasion on ultrasound suggestive of HCC, raises the intriguing possibility that CTC detection also may be associated with vascular invasion and poor prognosis in earlier stages of disease. This incidental finding, along with recent results of Schulze et al. and Sun et al. indicating prognostic value of CTC detection in patients with localized HCC $[14,15]$, suggest an important potential role for CTCs as a biomarker of occult vascular invasion, recurrence risk, and overall survival in patients with apparent localized disease undergoing evaluation for surgery or transplantation.

Our finding that EpCAM-positive CTCs are associated with high AFP and the presence of vascular invasion is 


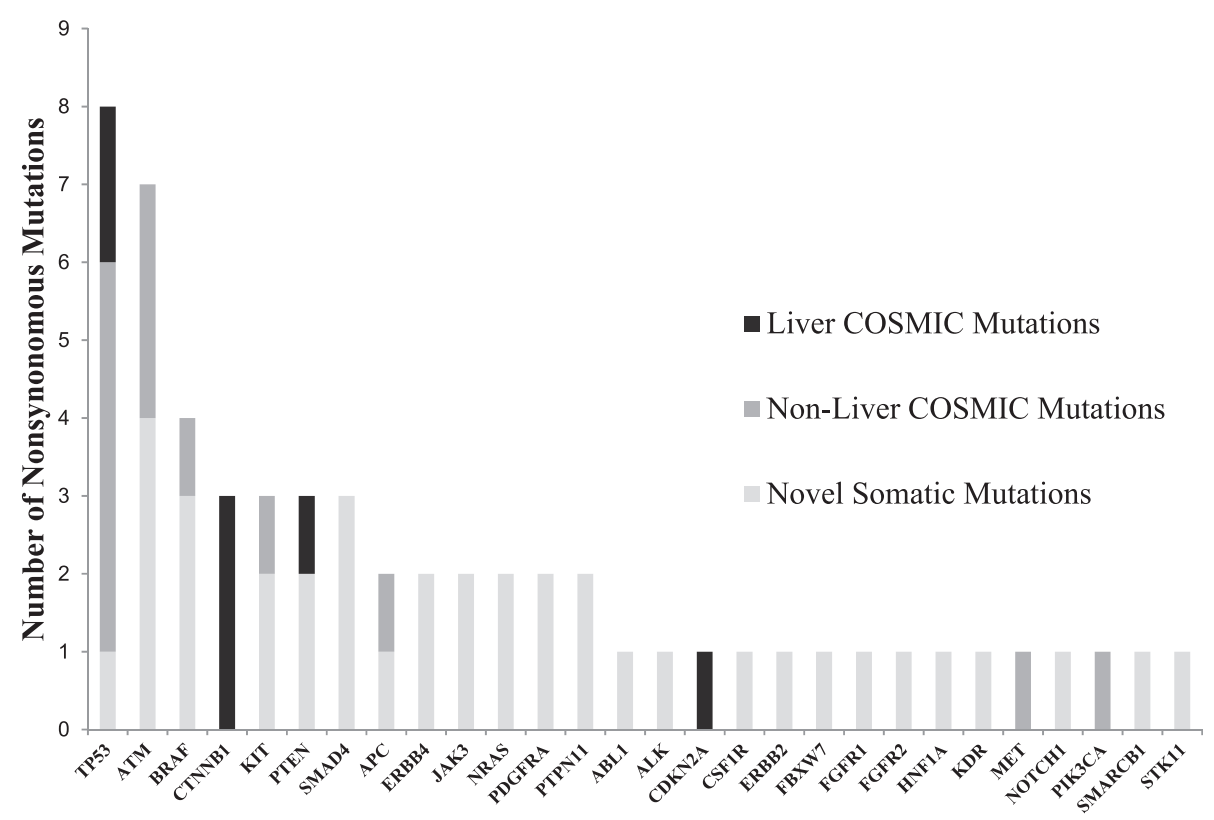

Figure 4 Summary of somatic, non-synonymous mutations. Occurring in CTC WGA DNA $(n=5)$ and/or tumor DNA $(n=6)$.

in keeping with the results of others $[14,15]$ which indicate that EpCAM-positive CTCs have biologic relevance as a diagnostic and prognostic biomarker in HCC. EpCAM expression and an EpCAM-positive gene expression signature are associated with poor differentiation, high AFP levels, and activation of Wnt- $\beta$-catenin signaling pathways [30-32]. EpCAM-positive HCC cells also express markers associated with cancer stem cells and the epithelial to mesenchymal transition, supporting a hypothesis that EPCAM enrichment identifies stemlike cells with potential for metastasis $[15,30,31,33]$.

A key unanswered question is whether EpCAM is the optimal marker for CTC enrichment in HCC. Unlike other epithelial tumor types which demonstrate nearly universal EpCAM expression [34], ЕpCAM is not expressed on mature hepatocytes and is expressed in only approximately $35 \%$ to $60 \%$ of HCC tumors by immunohistochemistry or PCR-based methods [30,31,35-37]. Thus, it is possible that non-EpCAM-expressing HCC cells exist in circulation and are undetectable by technologies employing EpCAM enrichment, which may account for our inability to detect CTCs in some of our HCC patients. Small series of non-EpCAM-based CTC isolation methods, such selection for the expression of asialoglycoprotein receptor or pancytokeratin or by cell size, suggest numerically higher incidence of detectable CTCs in metastatic HCC patients than has been reported with CellSearch, though the data are limited by small sample sizes and are not comparative $[16,17,38]$. Optimal CTC isolation and enrichment in $\mathrm{HCC}$ may require combining EpCAM with other markers.
Beyond using CTC detection and enumeration as a prognostic biomarker, however, CTCs offer a dynamic window into the evolution of metastatic disease. The advent of next-generation sequencing has revealed a remarkable degree of heterogeneity within individual tumors and between primary tumors and their metastases [39]. With increasingly sensitive and precise technologies for the detection and molecular profiling of rare cells, the genomic interrogation of CTCs may offer a powerful new tool to characterize, and someday to target, the dominant tumor subclones responsible for treatment resistance or metastatic progression. Heitzer et al. recently reported the first comprehensive genomic profiling of single CTCs using array comparative genomic hybridization and nextgeneration sequencing in a study of 37 patients with metastatic colorectal cancer [18]. Among the 6 patients with adequate $(>10)$ CTCs isolated for genomic profiling, concordance on copy number changes and characteristic driver mutations including PIK3CA, APC, and KRAS was shown, along with many additional mutations in the CTCs which were later found to be present at subclonal levels in the primary tumors by deep sequencing. Interestingly, heterogeneity was observed between CTCs isolated from the same patient at the same time-point.

This pilot study represents the first report of efficient isolation and next-generation sequencing of CTCs in HCC, to our knowledge. In this study, ion semiconductor next-generation sequencing showed a significantly higher proportion of targeted bases with at least 100x coverage depth among FFPE tumor and PBMC samples (87\%) compared to CTC-derived DNA samples 
(43\%) $(p<0.025)$. The disparate coverage depths according to sample type may be due in part to the use of an adaptor-ligation PCR WGA method which has been associated with allelic loss; alternate methods of amplification such as multiple displacement may mitigate this effect [40-42]. An alternate or contributory factor leading to the difference in allele frequency between sample types, as well as to the mutational disagreements between FFPE and CTC samples, may be the inherent heterogeneity of individual CTCs which were pooled for WGA from each patient [43]. WGA may also introduce low frequency variants by artifact $[40,41]$.

In our study, 86 variants were identified from CTC and FFPE tumor samples. One half of the variants were low frequency $(<10 \%)$ and derived predominantly from the CTC DNA samples. While again this finding could be due to coverage bias or artifact arising from WGA, these results are also consistent with the findings from Heitzer et al. in a colorectal cancer cohort [18], which suggest significant inter-CTC heterogeneity and could explain the prevalence of low-frequency variants arising from pooled DNA derived from multiple CTCs from an individual patient. Characteristic mutations associated with HCC (including TP53 and PTEN) were identified in CTC-derived DNA, consistent with tumor origin [44]. The overall sequencing accuracy in this study was demonstrated by several cases with available paired PBMC, CTC, and tumor DNA samples showing concordance on SNP calls, along with reproducibility of results in duplicate runs. A significant limitation of the exploratory sequencing in this pilot study, however, was its small sample size, along with the limited proportion of cases with paired CTC, FFPE tumor, and PBMC DNA available.

\section{Conclusions}

This study strongly supports that circulating epithelial cells are detectable in HCC patients, including via the CellSearch assay; and that these cells are EpCAMpositive tumor cells in circulation, rather than benign epithelial cells released in the setting of liver injury. These findings are based on significant CTC detection in HCC but not in NMLD cohorts, associations between CTC detection and HCC prognostic markers, and the demonstration of characteristic HCC mutations in DNA derived from purified CTCs. The significant association with macrovessel invasion and elevated AFP in this study, along with a trend towards poorer survival, indicate the potential value of CTC detection as a prognostic biomarker in metastatic HCC. Prospective analyses of CTCs in earlier stages of disease are warranted to determine surrogacy for vascular invasion in patients undergoing evaluation for surgery or liver transplantation. In parallel, we demonstrate that CTCs offer a source of noninvasive tumor DNA for next-generation sequencing and molecular profiling efforts in HCC. Future studies to determine the optimal CTC isolation technology, cut-points by assay and population, and methods for single-cell CTC molecular characterization are essential to develop CTCs as a clinical biomarker as well as a research tool in this grim, complex disease in urgent need of new biomarkers and therapeutic targets.

\section{Additional files}

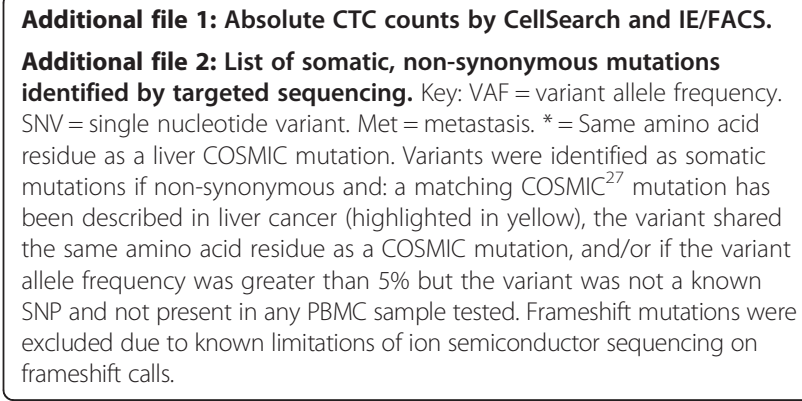

Competing interests

The authors declare that they have no competing interests.

\section{Authors' contributions}

RKK developed study concept, design, and protocol, consented and enrolled patients, managed and analyzed data, and wrote manuscript. MJ-MM performed CTC and WGA assays and contributed to data analysis and writing. TMB performed sequencing and analysis of sequencing data. EAC performed DNA extraction and contributed to study design, analysis, and writing. JH participated in study design and performed statistical analysis. NS performed DNA extraction. KE reviewed and marked pathology specimens for tumor content. RMM assisted in patient consent, blood specimen collection, and study coordination. BH, EMW, and FYY identified and consented control cases. APV participated in study design and data analysis. JWP participated in study design, developed IE/FACS assay, and contributed to data analysis and writing. All authors read and approved the final manuscript.

\section{Acknowledgments}

We acknowledge and deeply appreciate the patients who donated their specimens and time to participate in this biomarker study. The study was funded by a grant to RKK from the Mt. Zion Health Fund, University of California, San Francisco. Support for specimen processing was provided by The Bili Project Foundation, Inc. RKK's effort was funded in part by a Young Investigator Award (YIA) from the American Society of Clinical Oncology (ASCO) and by the NHGRI (R01HG007063, Pl: Phillips). KE was a Robert Black Fellow of the Damon Runyon Cancer Research Foundation (DRG-109-10) and is supported by the NCI/NIH (1K08CA172288-01A1). We thank Janet Scott, Eduardo Sosa, and Adam Foye for technical assistance in specimen processing.

\section{Author details}

${ }^{1}$ Helen Diller Family Comprehensive Cancer Center and The Liver Center, University of California San Francisco (UCSF), 550 16th St., Box 3211, San Francisco, CA 94143, USA. ${ }^{2}$ Helen Diller Family Comprehensive Cancer Center, UCSF, San Francisco, CA 94143, USA. ${ }^{3}$ Department of Molecular and Medical Genetics, Oregon Health Sciences University, 3181 SW Sam Jackson Park Road, Mail Code \#L103, Portland, OR 97239, USA. ${ }^{4}$ University of Vermont Medical Center, 89 Beaumont Ave., Burlington, VT 05405, USA. ${ }^{5}$ Department of Pathology, UCSF, 513 Parnassus Ave., San Francisco, CA 94143, USA. ${ }^{6}$ Division of Hepatology and Liver Transplant, UCSF, 513 Parnassus Ave., S-357, San Francisco, CA 94143, USA. ${ }^{7}$ Department of

Transplantation-Abdominal, UCSF, 513 Parnassus Ave., S-357, San Francisco, CA 94143, USA. ${ }^{8}$ Division of Hepatology and Liver Transplant and The Liver Center, UCSF, 513 Parnassus Ave., S-357, San Francisco, CA 94143, USA. 
Received: 27 May 2014 Accepted: 16 March 2015

Published online: 31 March 2015

\section{References}

1. Ferlay J, Shin HR, Bray F, Forman D, Mathers C, Parkin DM. Estimates of worldwide burden of cancer in 2008: GLOBOCAN 2008. Int J Cancer. 2010;127(12):2893-917.

2. Rodriguez-Peralvarez M, Luong TV, Andreana L, Meyer T, Dhillon AP, Burroughs AK. A systematic review of microvascular invasion in hepatocellular carcinoma: diagnostic and prognostic variability. Ann Surg Oncol. 2013;20(1):325-39.

3. Lim KC, Chow PK, Allen JC, Chia GS, Lim M, Cheow PC, et al. Microvascular invasion is a better predictor of tumor recurrence and overall survival following surgical resection for hepatocellular carcinoma compared to the Milan criteria. Ann Surg. 2011;254(1):108-13.

4. Bruix J, Sherman M. Management of hepatocellular carcinoma: an update. Hepatology. 2011;53(3):1020-2.

5. Mullhaupt B, Durand F, Roskams T, Dutkowski P, Heim M. Is tumor biopsy necessary? Liver Transpl. 2011;17 Suppl 2:S14-25.

6. Allard WJ, Matera J, Miller MC, Repollet M, Connelly MC, Rao C, et al. Tumor cells circulate in the peripheral blood of all major carcinomas but not in healthy subjects or patients with nonmalignant diseases. Clin Cancer Res. 2004;10(20):6897-904.

7. Miller MC, Doyle GV, Terstappen LW. Significance of circulating tumor cells detected by the cell search system in patients with metastatic breast colorectal and prostate cancer. J Oncol. 2010;2010:617421.

8. Cohen SJ, Punt CJ, lannotti N, Saidman BH, Sabbath KD, Gabrail NY, et al. Relationship of circulating tumor cells to tumor response, progression-free survival, and overall survival in patients with metastatic colorectal cancer. J Clin Oncol. 2008;26(19):3213-21.

9. Cohen SJ, Punt CJ, lannotti N, Saidman BH, Sabbath KD, Gabrail NY, et al. Prognostic significance of circulating tumor cells in patients with metastatic colorectal cancer. Ann Oncol. 2009;20(7):1223-9.

10. Cristofanilli M, Budd GT, Ellis MJ, Stopeck A, Matera J, Miller MC, et al. Circulating tumor cells, disease progression, and survival in metastatic breast cancer. N Engl J Med. 2004;351(8):781-91.

11. Liu MC, Shields PG, Warren RD, Cohen P, Wilkinson M, Ottaviano YL, et al. Circulating tumor cells: a useful predictor of treatment efficacy in metastatic breast cancer. J Clin Oncol. 2009;27(31):5153-9.

12. Magbanua MJ, Sosa EV, Scott JH, Simko J, Collins C, Pinkel D, et al. Isolation and genomic analysis of circulating tumor cells from castration resistant metastatic prostate cancer. BMC Cancer. 2012;12:78.

13. Okegawa T, Nutahara K, Higashihara E. Prognostic significance of circulating tumor cells in patients with hormone refractory prostate cancer. J Urol. 2009:181(3):1091-7.

14. Schulze K, Gasch C, Staufer K, Nashan B, Lohse AW, Pantel K, et al. Presence of EpCAM-positive circulating tumor cells as biomarker for systemic disease strongly correlates to survival in patients with hepatocellular carcinoma. Int J Cancer. 2013;133(9):2165-71.

15. Sun YF, Xu Y, Yang XR, Guo W, Zhang X, Qiu SJ, et al. Circulating stem cell-like epithelial cell adhesion molecule-positive tumor cells indicate poor prognosis of hepatocellular carcinoma after curative resection. Hepatology. 2013;57(4):1458-68.

16. Vona G, Estepa L, Beroud C, Damotte D, Capron F, Nalpas B, et al. Impact of cytomorphological detection of circulating tumor cells in patients with liver cancer. Hepatology. 2004;39(3):792-7.

17. Xu W, Cao L, Chen L, Li J, Zhang XF, Qian HH, et al. Isolation of circulating tumor cells in patients with hepatocellular carcinoma using a novel cell separation strategy. Clin Cancer Res. 2011;17(11):3783-93.

18. Heitzer E, Auer M, Gasch C, Pichler M, Ulz P, Hoffmann EM, et al. Complex tumor genomes inferred from single circulating tumor cells by array-CGH and next-generation sequencing. Cancer Res. 2013;73(10):2965-75.

19. Magbanua MJ, Sosa EV, Roy R, Eisenbud LE, Scott JH, Olshen A, et al. Genomic profiling of isolated circulating tumor cells from metastatic breast cancer patients. Cancer Res. 2013;73(1):30-40.

20. Ni X, Zhuo M, Su Z, Duan J, Gao Y, Wang Z, et al. Reproducible copy number variation patterns among single circulating tumor cells of lung cancer patients. Proc Natl Acad Sci U S A.

2013;110(52):21083-8.
21. Beadling C, Neff TL, Heinrich MC, Rhodes K, Thornton M, Leamon J, et al. Combining highly multiplexed PCR with semiconductor-based sequencing for rapid cancer genotyping. J Mol Diagn. 2013;15(2):171-6.

22. Liu L, Li Y, Li S, Hu N, He Y, Pong R, et al. Comparison of next-generation sequencing systems. J Biomed Biotechnol. 2012;2012:251364.

23. Magbanua MJ, Carey LA, DeLuca A, Hwang J, Scott JH, Rimawi MF, et al. Circulating tumor cell analysis in metastatic triple-negative breast cancers. Clin Cancer Res. 2015;21(5):1098-105. doi:10.1158/1078-0432.CCR-14-1948. Epub 2014 Dec 18

24. Magbanua MJ, Park JW. Isolation of circulating tumor cells by immunomagnetic enrichment and fluorescence-activated cell sorting (IE/FACS) for molecular profiling. Methods. 2013;64(2):114-8.

25. Barker DL, Hansen MS, Faruqi AF, Giannola D, Irsula OR, Lasken RS, et al. Two methods of whole-genome amplification enable accurate genotyping across a 2320-SNP linkage panel. Genome Res. 2004;14(5):901-7.

26. Ma WJ, Wang HY, Teng LS. Correlation analysis of preoperative serum alpha-fetoprotein (AFP) level and prognosis of hepatocellular carcinoma (HCC) after hepatectomy. World I Surg Oncol. 2013;11:212.

27. Tangkijvanich $P$, Anukulkarnkusol $N$, Suwangool $P$, Lertmaharit $S$, Hanvivatvong O, Kullavanijaya P, et al. Clinical characteristics and prognosis of hepatocellular carcinoma: analysis based on serum alpha-fetoprotein levels. J Clin Gastroenterol. 2000;31(4):302-8.

28. Forbes SA, Bindal N, Bamford S, Cole C, Kok CY, Beare D, et al. COSMIC: mining complete cancer genomes in the catalogue of somatic mutations in cancer. Nucleic Acids Res. 2011;39(Database issue):D945-50.

29. Kelley RK, Venook AP: Novel therapeutics in hepatocellular carcinoma. American Society of Clinical Oncology Educational Book 2013/ASCO American Society of Clinical Oncology Meeting 2013:137-142.

30. Yamashita T, Honda M, Nakamoto Y, Baba M, Nio K, Hara Y, et al. Discrete

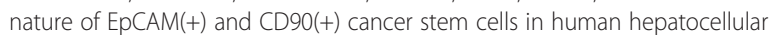
carcinoma. Hepatology. 2013;57(4):1484-97. doi:10.1002/hep.26168. Epub 2013 Jan 18.

31. Yamashita T, Forgues M, Wang W, Kim JW, Ye Q, Jia H, et al. EpCAM and alpha-fetoprotein expression defines novel prognostic subtypes of hepatocellular carcinoma. Cancer Res. 2008;68(5):1451-61.

32. Yamashita T, Budhu A, Forgues M, Wang XW. Activation of hepatic stem cell marker EpCAM by Wnt-beta-catenin signaling in hepatocellular carcinoma. Cancer Res. 2007:67(22):10831-9.

33. Yamashita T, Ji J, Budhu A, Forgues M, Yang W, Wang HY, et al. EpCAMpositive hepatocellular carcinoma cells are tumor-initiating cells with stem/ progenitor cell features. Gastroenterology. 2009;136(3):1012-24.

34. Went PT, Lugli A, Meier S, Bundi M, Mirlacher M, Sauter G, et al. Frequent EpCam protein expression in human carcinomas. Hum Pathol. 2004;35 (1):122-8.

35. Liang J, Ding T, Guo ZW, Yu XJ, Hu YZ, Zheng L, et al. Expression pattern of tumour-associated antigens in hepatocellular carcinoma: association with immune infiltration and disease progression. Br J Cancer. 2013;109(4):1031-9.

36. Zeng SS, Yamashita T, Kondo M, Nio K, Hayashi T, Hara Y, et al. The transcription factor SALL4 regulates stemness of EpCAM-positive hepatocellular carcinoma. J Hepatol. 2014;60(1):127-34.

37. de Boer CJ, van Krieken JH, Janssen-van Rhijn CM, Litvinov SV. Expression of Ep-CAM in normal, regenerating, metaplastic, and neoplastic liver. J Pathol. 1999;188(2):201-6.

38. Kelley RK, Nimeiri HS, Munster PN, Vergo MT, Huang Y, Li CM, et al. Temsirolimus combined with sorafenib in hepatocellular carcinoma: a phase I dose-finding trial with pharmacokinetic and biomarker correlates. Ann Oncol. 2013;24(7):1900-7.

39. Gerlinger M, Rowan AJ, Horswell S, Larkin J, Endesfelder D, Gronroos E, et al. Intratumor heterogeneity and branched evolution revealed by multiregion sequencing. N Engl J Med. 2012;366(10):883-92.

40. Park JW, Beaty TH, Boyce P, Scott AF, McIntosh I. Comparing wholegenome amplification methods and sources of biological samples for single-nucleotide polymorphism genotyping. Clin Chem. 2005;51(8):1520-3.

41. Stokes A, Drozdov I, Guerra E, Ouzounis CA, Warnakulasuriya S, Gleeson MJ, et al. Copy number and loss of heterozygosity detected by SNP array of formalin-fixed tissues using whole-genome amplification. PLoS One. 2011;6(9):e24503.

42. Swennenhuis JF, Reumers J, Thys K, Aerssens J, Terstappen LW. Efficiency of whole genome amplification of single circulating tumor cells enriched by Cell Search and sorted by FACS. Genome Med. 2013;5(11):106. 
43. Fujimoto A, Totoki Y, Abe T, Boroevich KA, Hosoda F, Nguyen HH, et al. Whole-genome sequencing of liver cancers identifies etiological influences on mutation patterns and recurrent mutations in chromatin regulators. Nat Genet. 2012;44(7):760-4.

44. Guichard C, Amaddeo G, Imbeaud S, Ladeiro Y, Pelletier L, Maad IB, et al. Integrated analysis of somatic mutations and focal copy-number changes identifies key genes and pathways in hepatocellular carcinoma. Nat Genet. 2012;44(6):694-8.

Submit your next manuscript to BioMed Central and take full advantage of:

- Convenient online submission

- Thorough peer review

- No space constraints or color figure charges

- Immediate publication on acceptance

- Inclusion in PubMed, CAS, Scopus and Google Scholar

- Research which is freely available for redistribution 